\title{
Evaluation of open search methods based on theoretical mass spectra comparison
}

\author{
Albane Lysiak ${ }^{1,3+}$, Guillaume Fertin ${ }^{{ }^{* *}}$ (D) Géraldine Jean $^{1+}$ and Dominique Tessier ${ }^{2,3+}$
}

From Computational Intelligence methods for Bioinformatics and Biostatistics Bergamo, Italy. 4-6 September 2019

\author{
*Correspondence: \\ guillaume.fertin@Is2n.fr \\ ${ }^{\dagger}$ Albane Lysiak, Guillaume \\ Fertin, Géraldine Jean and \\ Dominique Tessier have \\ contributed equally to this \\ work \\ ${ }^{1}$ CNRS, LS2N, Université de \\ Nantes, 44000 Nantes, France \\ Full list of author information \\ is available at the end of the \\ article
}

\begin{abstract}
Background: Mass spectrometry remains the privileged method to characterize proteins. Nevertheless, most of the spectra generated by an experiment remain unidentified after their analysis, mostly because of the modifications they carry. Open Modification Search (OMS) methods offer a promising answer to this problem. However, assessing the quality of OMS identifications remains a difficult task.
\end{abstract}

Methods: Aiming at better understanding the relationship between (1) similarity of pairs of spectra provided by OMS methods and (2) relevance of their corresponding peptide sequences, we used a dataset composed of theoretical spectra only, on which we applied two OMS strategies. We also introduced two appropriately defined measures for evaluating the above mentioned spectra/sequence relevance in this context: one is a color classification representing the level of difficulty to retrieve the proper sequence of the peptide that generated the identified spectrum ; the other, called LIPR, is the proportion of common masses, in a given Peptide Spectrum Match (PSM), that represent dissimilar sequences. These two measures were also considered in conjunction with the False Discovery Rate (FDR).

Results: According to our measures, the strategy that selects the best candidate by taking the mass difference between two spectra into account yields better quality results. Besides, although the FDR remains an interesting indicator in OMS methods (as shown by LIPR), it is questionable: indeed, our color classification shows that a non negligible proportion of relevant spectra/sequence interpretations corresponds to PSMs coming from the decoy database.

Conclusions: The three above mentioned measures allowed us to clearly determine which of the two studied OMS strategies outperformed the other, both in terms of number of identifications and of accuracy of these identifications. Even though quality evaluation of PSMs in OMS methods remains challenging, the study of theoretical spectra is a favorable framework for going further in this direction.

Keywords: Mass spectrometry, Open Modification Search, Peptide identification, Blind search author(s) and the source, provide a link to the Creative Commons licence, and indicate if changes were made. The images or other third party material in this article are included in the article's Creative Commons licence, unless indicated otherwise in a credit line to the material. If material is not included in the article's Creative Commons licence and your intended use is not permitted by statutory regulation or exceeds the permitted use, you will need to obtain permission directly from the copyright holder. To view a copy of this licence, visit http:// creativecommons.org/licenses/by/4.0/. The Creative Commons Public Domain Dedication waiver (http://creativecommons.org/publi cdomain/zero/1.0/) applies to the data made available in this article, unless otherwise stated in a credit line to the data. 


\section{Background}

Proteomics is at the core of various studies that aim at understanding the complexity of life. In particular, one of the objectives is to discover all the modifications that can affect proteins, and possibly result in a modulation or a total change of their cellular functions $[1,2]$. Mass spectrometry in tandem MS mode (MS/MS) is the most powerful method to identify proteins and characterize their modifications on a large scale. However, it remains frustrating to observe that, in spite of an abundant literature on the subject, most of the spectra generated by this analytical technique-namely, tens of thousands of spectra per hour of analysis-are left unidentified after their analysis by a dedicated software. The reason behind this low rate of identification is likely due to the large proportion of spectra generated from proteins carrying modifications [3]. Software usually infer the identification of an experimental spectrum from its similarity to reference spectra. When a peptide carries a modification, its mass is by nature modified. This mass modification prevents its identification by conventional methods, which compare each experimental spectrum with only a restricted set of reference spectra that approximately share the same mass in order to avoid excessive runtime. Some known modifications can be included in the modeling of reference spectra, but their number must remain low to circumscribe the search space.

In 2015, the study conducted by Chick et al. [4] renewed the interest in so-called Open Modification Search (OMS) methods known since 2005 [5, 6], with the promise of revealing unexpected modifications which would have been lost otherwise, and consequently gaining better rates of spectra identifications. OMS methods compare each experimental spectrum to all the reference spectra representing a proteome. Then, whereas conventional methods, by definition, try to identify pairs of spectra (experimental vs reference) that are supposed to represent the same chemical compound (i.e., an ideal reference spectrum is matched to its imperfect experimental counterpart), OMS methods allow matches between similar spectra that represent distinct chemical compounds with unequal masses. As a result, this comparison produces a list of PSMs (Peptide Spectrum Matches) per experimental spectrum, and a non-zero mass difference $\Delta m$ between the experimental spectrum and its associated peptide is assumed to be due to one or several modifications that differentiate them. Most of the time, only one PSM per experimental spectrum is reported: each experimental spectrum is associated to the most similar reference spectrum. Many scores exist to evaluate the similarity between two spectra, which all take into account, at a certain level, the number of peaks (or, equivalently, of masses) that are shared by the two spectra, a number called shared peaks count (SPC). Once the complete list of PSMs is sorted by score, a threshold based on a measure of statistical significance determines which PSMs are validated according to a false discovery rate (FDR) [7].

Since 2015, several very rapid OMS methods have been developed. For a few of them, the reference spectra come from consensus templates already observed, identified and stored in spectral librairies [8-11]; for others, the reference spectra, so-called theoretical spectra in that case, are generated from a protein database by simulating an ideal fragmentation of peptides (see Fig. 1) [12-14].

Besides the comparison of spectral pairs, some methods also associate pairs of spectra when they share sequence tags [15-17]. These methods have improved the identification 


\begin{tabular}{|c|c|c|c|c|c|c|}
\hline Ion & Sequence & Mass (Da) & & Ion & Sequence & Mass (Da) \\
\hline b1 & $\mathrm{E}-\mathrm{H}^{+}$ & 130.0499 & & y1 & $\mathrm{H}_{2}{ }^{+}-\mathrm{K}-\mathrm{OH}$ & 147.1128 \\
\hline b2 & $\mathrm{EA}-\mathrm{H}^{+}$ & 201.087 & $y$ ions & $y^{2}$ & $\mathrm{H}_{2}^{+}-\mathrm{KE}-\mathrm{OH}$ & 276.1554 \\
\hline b3 & $\mathrm{EAE}-\mathrm{H}^{+}$ & 330.1296 & & y3 & $\mathrm{H}_{2}{ }^{+}-\mathrm{KES}-\mathrm{OH}$ & 363.1874 \\
\hline b4 & EAEI-H ${ }^{+}$ & 443.2136 & HS-EFK T $^{\mathrm{OH}}$ & $y^{4}$ & $\mathrm{H}_{2}{ }^{+}-\mathrm{KESI}-\mathrm{OH}$ & 476.2715 \\
\hline b5 & EAEIS- $\mathrm{H}^{+}$ & 530.2457 & & y5 & $\mathrm{H}_{2}{ }^{+}-\mathrm{KESIE}-\mathrm{OH}$ & 605.3141 \\
\hline b6 & EAEISE-H ${ }^{+}$ & 659.2883 & & y6 & $\mathrm{H}_{2}^{+}-\mathrm{KESIEA}-\mathrm{OH}$ & 676.3512 \\
\hline b7 & EAEISEK- ${ }^{+}$ & 787.3832 & & y7 & $\mathrm{H}_{2}{ }^{+}$-KESIEAE-OH & 805.3938 \\
\hline
\end{tabular}

Fig. 1 Example of in silico generation of a theoretical spectrum. The theoretical spectrum of peptide EAEISEK is composed of masses of the two main types of ions obtained by mass spectrometry: the $b$ series (blue table) and $y$ series (red table). A $b$-ion contains the $\mathrm{N}$-terminal part of the peptide and is numbered according to the peptide sequence, from left to right. A $y$-ion contains the C-terminal part of the peptide and is numbered backwards according to the peptide sequence, from right to left. Masses are given in Daltons

rate and as much as an almost twofold increase in the rate of identified spectra has been reported $[15,18]$. However, despite the scientific relevance of better spectra identifications, OMS methods are still underused, notably because their reliability remains debated. It is therefore important to better describe the advantages and limitations of these methods. Because all OMS methods have in common that at some point, they must decide wether or not to use the information of $\Delta m$ within a PSM to infer a better identification, we focused our study on a thorough undertanding of two widely spread strategies to determine the best PSM for each experimental spectrum. In the first strategy (called Strategy 1 in this paper), the best PSM is chosen according to a score that does not take the $\Delta m$ into account. The second strategy (called Strategy2) tries to improve the alignment-and thus the score (Fig. 2) —of all the PSMs returned for a given experimental spectrum according to $\Delta m$ before the choice of the best PSM. In order to determine the most efficient strategy, a prerequisite was to be able to implement both strategies using the same software, which implies the availability of very efficient spectra comparison and alignment algorithms. The SpecOMS software [14], which we have previously developed, fulfills these conditions.

To compare in-depth the limits of each strategy, we decided to ground this study using the theoretical spectra derived from the human proteome, considering successively each theoretical spectrum in the role of an experimental spectrum. In such a way, we eliminate the inherent identification difficulties due to the imperfection of experimental spectra (noise, missing peaks, etc.) and concentrated on the benefits of each strategy. Consequently, PSMs with $\Delta m \neq 0$ can only be explained by differences in terms of amino acids, namely insertions, deletions and/or substitutions of one or several amino acids. Note that any modification (whether it is a post-translational modification (PTM) or a sequence modification) induces the same number of peak shifts in a spectrum. Every PSM matching a peptide to itself was considered irrelevant and consequently forbidden. Thus, we challenged the two above strategies to discover many diverse modifications, while keeping a certain proximity between peptides (since they arise from the same proteome). As conventional methods do, many OMS methods estimate the FDR of their results with a target/decoy approach. We also used this approach to compare both strategies, even though it is still unclear whether or not this method underestimates the 


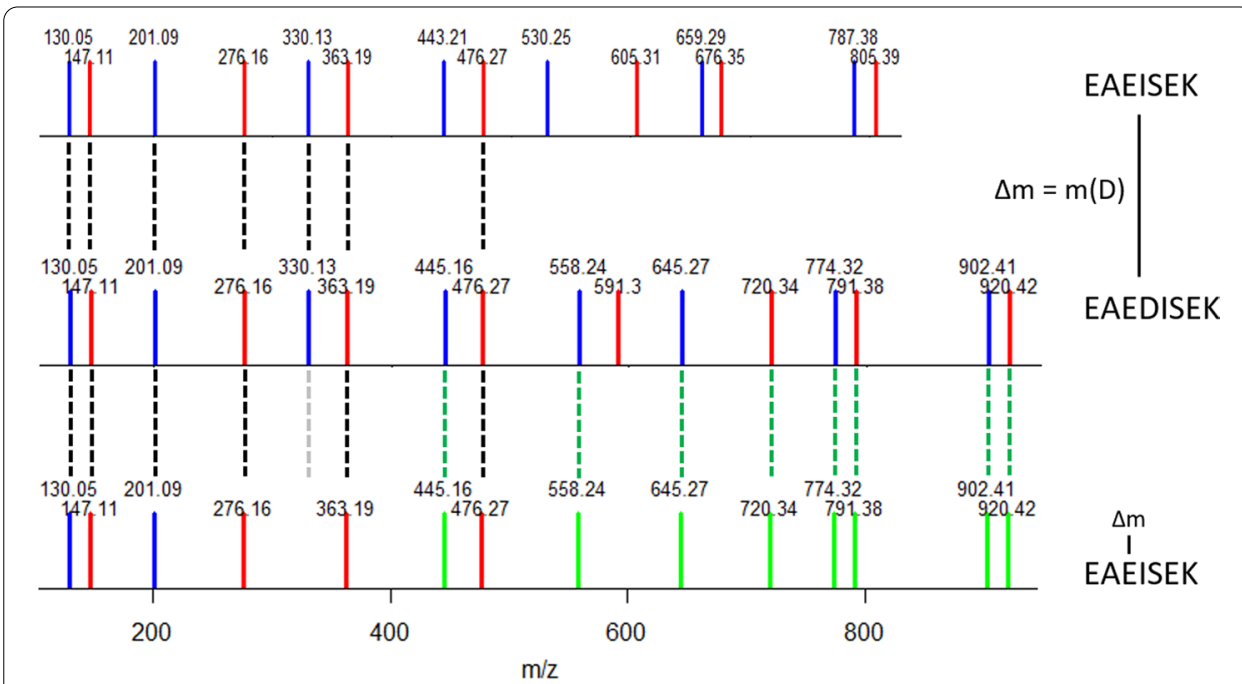

Fig. 2 MS/MS spectra matches and their peptide sequences. $b$-ions are displayed in blue, $y$-ions in red, and matches between spectra in dashed lines. Intensities of all peaks are set to an arbitrary unit value in theoretical spectra. The EAEDISEK MS/MS spectrum (in the middle) shares 7 masses (black dashed lines) with the native EAEISEK spectrum (above). After a shift of $\Delta m$ at position 3 in EAEISEK (below), 8 new masses match with EAEDISEK (as shown in green), and one match is removed (grey dashed line). The SPC is then improved from 7 (raw SPC) to 14 (shift SPC)

incorrect identifications $[19,20]$. That is why, we propose two additional measures of the PSM characteristics to evaluate their quality and compare the strategies.

\section{Results}

We successively implemented Strategy1 and Strategy2 (see "Methods" section) to compare all the theoretical spectra generated from the human proteome $(572,063$ spectra) against a database merging the target and decoy human proteins $(1,148,608$ spectra). To denote unambiguously the role that each theoretical spectrum can alternately play, we call it bait when it plays the role of an experimental spectrum and hit when it represents the theoretical spectrum modeled from the protein database. By extension, we also designate by bait and hit the peptides that generated respectively these theoretical spectra. Any pair (bait,hit) having an SPC greater than or equal to our initial threshold 7 (see "Methods" section) is called a candidate PSM. Any PSM returned by our software will be called best PSM. On the one hand, Strategy1 selects the best hit according to the raw SPC score, computed without taking into account the observed $\triangle m$. On the other hand, Strategy 2 iteratively tests, on each possible amino acid and at the $\mathrm{N}$-terminal and C-terminal sides of the peptide, whether a modification of mass $\triangle m$ could improve the alignment between the two spectra [21]. In this latter case, a new score shift SPC is computed, which corresponds to the number of shared masses after realignement, and the best PSM is selected according to this new score.

The results are summarized in Tables 1 and 2. In each of the two strategies, SpecOMS reported 455,404 best PSMs structured in the form of tuples (bait,hit,SPC, $\Delta m$ ), where (a) hit is the best candidate for bait (depending on the selected strategy), (b) SPC is the number of shared masses between bait and hit (according to raw SPC in Strategy1 and to 
Table 1 Number of PSMs obtained by Strategy1 according to raw SPC

\begin{tabular}{|c|c|c|c|c|c|c|c|c|c|}
\hline \multirow[t]{2}{*}{ Min raw SPC } & \multicolumn{2}{|l|}{$\Delta m=0$} & \multicolumn{2}{|c|}{$\Delta m<0$} & \multicolumn{2}{|l|}{$\Delta m>0$} & \multicolumn{3}{|l|}{ Total } \\
\hline & \#target & \#decoy & \#target & \#decoy & \#target & \#decoy & \#target & \#decoy & FDR(\%) \\
\hline 7 & 71,852 & 87,705 & 88,895 & 39,432 & 107,842 & 59,678 & 268,589 & 186,815 & 41.02 \\
\hline 8 & 71,852 & 87,705 & 55,874 & 12,352 & 61,225 & 19,550 & 188,951 & 119,607 & 38.76 \\
\hline 9 & 32,972 & 32,740 & 41,378 & 3874 & 40,085 & 4822 & 114,435 & 41,436 & 26.58 \\
\hline 10 & 32,970 & 32,739 & 31,973 & 868 & 30,245 & 1026 & 95,188 & 34,633 & 26.68 \\
\hline 11 & 10,918 & 11,209 & 26,095 & 310 & 24,547 & 305 & 61,560 & 11,824 & 16.11 \\
\hline 12 & 10,918 & 11,208 & 21,553 & 112 & 20,313 & 94 & 52,784 & 11,414 & 17.78 \\
\hline 13 & 2571 & 1180 & 17,893 & 46 & 16,939 & 34 & 37,403 & 1260 & 3.26 \\
\hline 14 & 2571 & 1180 & 14,955 & 22 & 14,243 & 17 & 31,769 & 1219 & 3.7 \\
\hline 15 & 1137 & 380 & 12,352 & 9 & 11,818 & 9 & 25,307 & 398 & 1.55 \\
\hline 16 & 1137 & 380 & 10,239 & 6 & 9801 & 5 & 21,177 & 391 & 1.81 \\
\hline 17 & 672 & 61 & 8403 & 5 & 8085 & 4 & 17,160 & 70 & 0.41 \\
\hline 18 & 672 & 61 & 6907 & 4 & 6662 & 3 & 14,241 & 68 & 0.48 \\
\hline 19 & 495 & 49 & 5590 & 2 & 5413 & 1 & 11,498 & 52 & 0.45 \\
\hline 20 & 495 & 49 & 4557 & 1 & 4415 & 1 & 9467 & 51 & 0.54 \\
\hline
\end{tabular}

The best PSMs provided by SpecOMS are separated into three groups based on $\Delta m$, and subdivided in each group by the origin of the hit (target or decoy database). An FDR $<1 \%$ is reached whenever raw SPC is greater than or equal to 17 (row in bold)

Table 2 Number of PSMs obtained by Strategy2 according to shift SPC

\begin{tabular}{|c|c|c|c|c|c|c|c|c|c|}
\hline \multirow[t]{2}{*}{ Min shift SPC } & \multicolumn{2}{|l|}{$\Delta m=0$} & \multicolumn{2}{|l|}{$\Delta m<0$} & \multicolumn{2}{|l|}{$\Delta m>0$} & \multicolumn{3}{|l|}{ Total } \\
\hline & \#target & \#decoy & \#target & \#decoy & \#target & \#decoy & \#target & \#decoy & FDR(\%) \\
\hline 7 & 14,207 & 17,401 & 145,801 & 86,218 & 122,479 & 69,298 & 282,487 & 17,2917 & 37.97 \\
\hline 9 & 5132 & 4787 & 145,756 & 86,183 & 122,465 & 69,287 & 273,353 & 160,257 & 36.96 \\
\hline 11 & 1944 & 1793 & 144,795 & 85,247 & 121,833 & 68,671 & 268,572 & 155,711 & 36.7 \\
\hline 13 & 721 & 343 & 139,895 & 80,327 & 119,006 & 65,901 & 259,622 & 146,571 & 36.08 \\
\hline 15 & 496 & 187 & 116,674 & 61,154 & 110,457 & 58,923 & 227,627 & 120,264 & 34.57 \\
\hline 17 & 341 & 42 & 58,584 & 130,53 & 55,297 & 130,52 & 114,222 & 26,147 & 18.63 \\
\hline 19 & 250 & 36 & 37,840 & 1681 & 35,439 & 1647 & 73,529 & 3364 & 4.37 \\
\hline 21 & 201 & 12 & 29,675 & 271 & 27,908 & 251 & 57,784 & 534 & 0.92 \\
\hline 23 & 160 & 10 & 24,262 & 87 & 22,881 & 82 & 47,303 & 179 & 0.38 \\
\hline 25 & 131 & 5 & 20,036 & 35 & 18,910 & 36 & 39,077 & 76 & 0.19 \\
\hline 27 & 108 & 4 & 16,686 & 18 & 15,792 & 14 & 32,586 & 36 & 0.11 \\
\hline 29 & 92 & 2 & 13,835 & 12 & 13,120 & 9 & 27,047 & 23 & 0.08 \\
\hline 31 & 67 & 2 & 11,345 & 4 & 10,801 & 5 & 22,213 & 11 & 0.05 \\
\hline 33 & 54 & 0 & 9314 & 2 & 8888 & 3 & 18,256 & 5 & 0.03 \\
\hline 35 & 44 & 0 & 7608 & 2 & 7296 & 2 & 14,948 & 4 & 0.03 \\
\hline 37 & 39 & 0 & 6231 & 2 & 5989 & 2 & 12,259 & 4 & 0.03 \\
\hline 39 & 17 & 0 & 5008 & 1 & 4832 & 1 & 9857 & 2 & 0.02 \\
\hline
\end{tabular}

The best PSMs provided by SpecOMS are separated into three groups based on $\Delta m$, and subdivided in each group by the origin of the hit (target or decoy database). An FDR $<1 \%$ is reached whenever shift SPC is greater than or equal to 21 (row in bold). Only results for odd minimum shift SPC are displayed here: indeed, shift SPC is always even, since a match always involves both $a b$-ion and a $y$-ion after realignment. The results we obtain are thus the same for consecutive odd and even shift SPC (except for a few exceptions due to overlapping masses)

shift SPC in Strategy2), (c) a mass difference of $\Delta m$ (expressed in Daltons) exists between bait and hit-more precisely $\Delta m$ is equal to mass of bait minus mass of hit. 
About $80 \%$ of the 572,063 tryptic peptides from the human proteome share at least 7 peaks with any other peptide, and about $23 \%$ of them share at least 10 peaks (target or decoy).

Since the number of best PSMs obtained by SpecOMS only depends on the number of pairs of spectra that initially share at least 7 masses ( 7 being the threshold parameter chosen in this study, see "Methods" section), the number of best PSMs remains identical in both strategies. By contrast, Strategy1 and Strategy2 provide dissimilar sets of best PSMs, that we respectively named $\mathrm{PSM}_{1}$ and $\mathrm{PSM}_{2}$ (see Fig. 6, "Methods" section). About 37\% of the PSMs (167,291 baits) differ between both strategies when the initial SPC threshold is set to 7. We separated $\mathrm{PSM}_{1}$ and $\mathrm{PSM}_{2}$ into 3 groups according to $\Delta m$ : $G_{1}$ is the group of PSMs such that $\Delta m=0$ (i.e., the bait and the hit have the same mass), $G_{2}$ is the group such that $\Delta m>0$ (the mass of the bait exceeds the mass of the hit) and $G_{3}$ is the group such that $\Delta m<0$ (the mass of the hit exceeds the mass of the bait). Note that when $\Delta m \neq 0$ in Strategy2, the score of the corresponding PSMs is likely to increase, while the score of PSMs with $\Delta m=0$ remains unchanged (since no realignment is possible). As a result, many candidate PSMs selected with $\Delta m=0$ in Strategy 1 has been overpassed by PSMs associated to $\Delta m \neq 0$ in Strategy 2 (127,949 PSMs). Next, we evaluated the confidence of the results with the typical target-decoy approach [7] and compute the minimum similarity score that guarantees a FDR usually accepted by users. Respectively to a FDR $<1 \%$, Strategy 1 validates 17,160 PSMs with a minimal raw SPC of 17 (i.e. considering best PSMs for which raw $S P C \geq 17$ ), while Strategy 2 validates 57,784 PSMs with a minimal shift SPC of 21 (i.e. considering best PSMs for which shift SPC $\geq 21$ ). Strategy 2 recruits more than three times more PSMs than Strategy1 so, we can conclude that Strategy 2 behaves better than Strategy1 according to the number of validated PSMs. Thus, $3 \%$ to $10 \%$ of the theoretical spectra have at least one "neighbor peptide" that shares a sufficient number of masses so that their similarity is not estimated to be considered as the result of chance. It must be remembered that a hit is supposed to directly identify a bait for PSMs with $\Delta m=0$. We know that it cannot be the case in our dataset, since we set SpecOMS so as to forbid PSMs involving the same peptide. Indeed, very few PSMs were validated by the target-decoy method in $G_{1}$ (672 PSMS with Strategy1, 201 PSMs with Strategy2), which is consistent with the composition of this group. On the opposite, the $G_{2}$ and $G_{3}$ groups represent most of the validated PSMs, but one may wonder to what extent the information given by these PSMs is enough to restore the correct amino acid sequence of the baits-a sine qua non condition to consider a posteriori an identification as correct.

To answer this question, we proposed new criteria to measure the quality of identifications. Knowing exactly which peptide generated each bait, we should be able to accurately assess the effectiveness of each strategy. Firstly, we defined a new classification that reflects the level of difficulty for a user or software to retrieve the bait sequence using both the hit sequence, the value of $\Delta m$ and the shift location. PSMs with $\Delta m \neq 0$ can only be explained by differences in terms of amino acid sequences. Then, by applying one or several editing operations (insertion(s), deletion(s), and/or substitution(s)), the hit can always be transformed into the bait. Given a PSM, if the bait can be deduced from the hit without any ambiguity in just one editing operation specified by $\Delta m$, we classified this PSM as Green. However, when $\Delta m$ corresponds 
to an insertion of several amino acids, this is not enough to reconstruct the sequence of the bait without ambiguity-several permutations of amino acids can lead to the same $\Delta m$. In this case, we classified the PSM as Orange. We classified all other PSMs as Red (see "Methods" section). As a result, the Red class contains a large variety of PSMs: those that require several to many editing operations to transform the bait into the hit (in other words, bait and hit sequences are very dissimilar), but also those whose sequences may be close, but that contain permutations of amino acids that are difficult to infer from $\Delta m$. In particular, PSMs with $\Delta m=0$ are obviously classified as Red, because the hit and the bait are different and no realignment is possible. An illustration of the color classification containing more examples is given in Fig. 3.

Secondly, we introduced a new feature, which we call the Low Information Peaks Rate (LIPR), that measures, for a given PSM, the proportion of masses that are shared by two spectra but correspond to different amino acid sequences (see "Methods" section). In summary, the higher this value, the less sequence information the PSM carries, in the sense that proportionally many shared peaks correspond to distinct amino acid sequences.

We present the distributions of the sets $\mathrm{PSM}_{1}$ and $\mathrm{PSM}_{2}$ obtained respectively by Strategy 1 and Strategy 2 among the 3 color classes, as well as the evaluation of the LIPR feature, in Figs. 4 and 5 . It can be seen that, globally, both strategies behave in a similar fashion, but at $1 \%$ FDR, Strategy 2 validates roughly three times more Green PSMs than Strategy1 (27,211 vs 9153). Thus, at first glance, the number of additional identifications obtained by Strategy 2 (compared to Strategy1) does not come at the cost of a deterioration of the quality of the results.

In terms of LIPR, it can be noted that its average value is higher for $\mathrm{PSM}_{1}(38.5 \%$ for $\mathrm{PSM}_{1}$ vs $22.97 \%$ for $\mathrm{PSM}_{2}$ ). Generally speaking, in both strategies the LIPR decreases

\begin{tabular}{|c|l|l|l|l|}
\hline \multicolumn{1}{|c|}{ Bait } & \multicolumn{1}{|c|}{ Hit } & \multicolumn{1}{c|}{$\Delta m(\mathrm{Da})$} & $\begin{array}{c}\text { Shift } \\
\text { location }\end{array}$ & Interpretation : from the hit to the bait \\
\hline TSSDSISR & TSDSISR & $\mathbf{8 7 . 0 3 2 0}$ & $\mathbf{3}$ & TSDSISR + S = TSSDSISR \\
\hline YQEFQNR & EPPNPEYQEFQNR & -663.2864 & 6 & EPPNPEYQEFQNR - EPPNPE = YQEFQNR \\
\hline ETVHIPGAR & ETIPGAR & 236.1273 & 2 & ETIPGAR + $\Delta \mathrm{m}=\mathrm{ET}-\Delta \mathrm{m}$-IPGAR \\
\hline VISPEDGK & VIESPDGK & $\mathbf{0}$ & $/$ & $/$ \\
\hline VCASIAQK & VTIQCQK & $\mathbf{0}$ & $/$ & $/$ \\
\hline WFSIYDQR & FWSIQDYFR & -147.0684 & $/$ & $/$ \\
\hline
\end{tabular}

Fig. 3 Illustration of the Green/Orange/Red classification of PSMs. The first two rows present PSMs with a bait unambiguously deductible from the information given by the PSM. Such PSMs are classified as Green. In the first example, $\Delta m$ corresponds to the mass of $S$, which can thus be added in the hit at the given location to retrieve the bait. In the second example, the absolute value of $\Delta m$ corresponds to the mass of EPPNPE, which can be deleted from the hit to retrieve the bait. In the third row, $\Delta m$ can correspond to two possible amino acid sequences (VH or HV). Such PSM is thus classified as Orange. In the last three rows, transforming hit into bait is more difficult because there is too much ambiguity, although sequences may be close as in the first red row. In all cases, such PSMs are classified as Red 

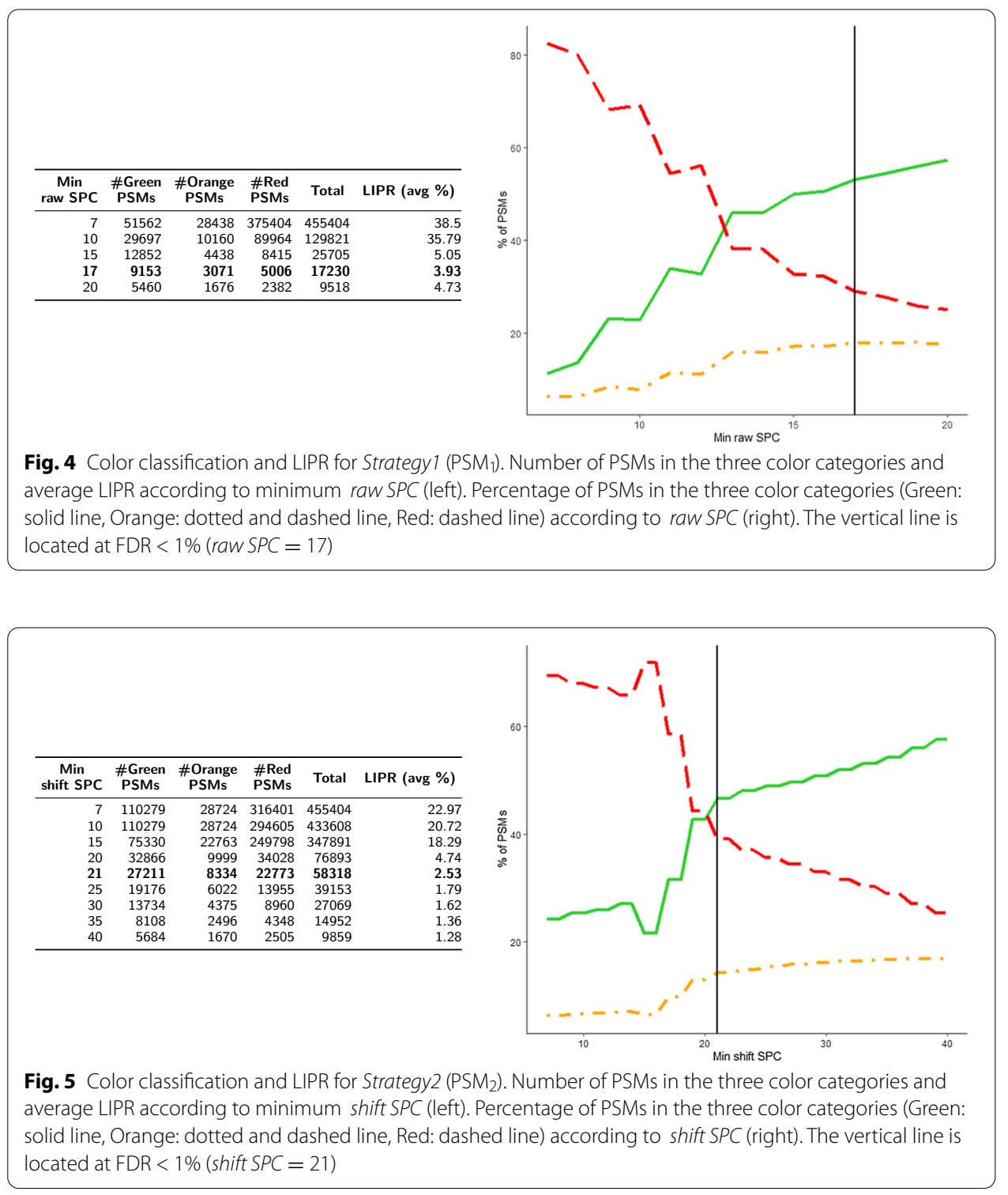

when the minimum SPC increases. At a 1\% FDR threshold, results are quite similar for both strategies (3.93\% for $\mathrm{PSM}_{1}$ vs $2.53 \%$ for $\mathrm{PSM}_{2}$ ).

A strong difference concerning LIPR appears between target and decoy PSMs, and this phenomenon is present in both strategies (results not shown). The average LIPR of target PSMs in $\mathrm{PSM}_{1}$ is $31 \%$, whereas it reaches $49 \%$ in decoy PSMs. This difference increases with the minimum SPC that is observed, and the LIPR suddenly drops near the 1\% FDR threshold ; for instance, for raw SPC $\geq 15$ in $\mathrm{PSM}_{1}$, the average LIPR of decoy PSMs is $64 \%$ whereas it is $4 \%$ for target PSMs. The LIPR can thus be seen as a way to capture the "randomness" of decoy PSMs, for which matches between peaks that correspond to different sequences are much more common than in target PSMs, especially for PSMs with high SPC values.

To highlight the differences between the PSMs provided by each strategy, we isolated PSMs specific to Strategy1 (a set of PSMs called $\mathrm{SS}_{1}$ ) from PSMs specific to Strategy2 (a 
set of PSMs called $\mathrm{SS}_{2}$ ). Since $\mathrm{SS}_{1}$ and $\mathrm{SS}_{2}$ are exactly what differentiates Strategy 1 from Strategy2, highlighting the main differences (or similarities) between these two sets is particularly informative. It should also be noted that this comparison can only be done at $\mathrm{SPC}=7$, thus with the whole set of (specific) PSMs, since raw SPC and shift SPC are by definition distinct scores.

The number of PSMs in each color class, as well as the average LIPR for $\mathrm{SS}_{1}$ and $\mathrm{SS}_{2}$ are displayed in Table 3. We can clearly see that Strategy2 contains many more PSMs classified as Green, compared to Strategy1 (more than 16 times more). The average LIPR is also much higher in $\mathrm{SS}_{1}(61.7 \%)$ than in $\mathrm{SS}_{2}(19.44 \%)$.

The results we obtain concerning the color classification and LIPR can be interpreted in two different manners. First, they can be considered independently of the FDR, based on the argument that the FDR is a measure that remains debated in OMS methods. Based on the results presented in Table 3, it can be seen that Strategy 2 clearly outperforms Strategy1. Another way to consider these two indicators is to compare them taking the FDR into account (namely, at an FDR less than 1\%). In that case (see Figs. 4 and 5) if Strategy 2 still outperforms Strategy1 in terms of number of PSMs (roughly 3 times more Green or Orange PSMs in Strategy2 than in Strategy1), the results in terms of percentage are in favour of Strategy1 (70.9\% of the PSMs are Green or Orange in Strategy1 vs $60.9 \%$ for Strategy2). However, the fact that our color classification marks as Red proportionally more PSMs from Strategy 2 than from Strategy 1 must be put into perspective with a lower LIPR value associated to Strategy2, indicating a close proximity between the bait and the hit. So, a large number of these Red PSMs are likely due to a very small number of editing operations like permutations in sequences. Then, a deeper analysis of the Red category should convert, with minimal additional computational efforts, many Red PSMs into Green or Orange PSMs. Besides, we can note the high proportion of PSMs from the human proteome classified as Green when $\mathrm{SPC} \geq 7$. If all the hits associated to those Green PSMs came from the target database, it would be come as very good news because these PSMs refer to peptides that differ by unambiguous editing operations from their "closest neighbor" (from $11 \%$ in Strategy1 to $24 \%$ in Strategy2). Since it is not the case, it means that the decoy database is not only constituted of "incorrect sequences" as it should be. Then, it is clear that for both strategies, the presence of many Green PSMs in the decoy database hinders the identification of the baits.

\section{Discussion}

In this work, we aimed at comparing two OMS strategies that frequently appear in recently published OMS methods. Evaluating the respective performances of the two studied strategies has been conducted through three different measures: along with the FDR, we introduced two ways of interpreting the PSMs: the first one is a color

Table 3 Average LIPR and distribution of PSMs in results specific to PSM $1\left(S_{1}\right)$ and specific to PSM 2 $\left(\mathrm{SS}_{2}\right)$ in the three color categories

\begin{tabular}{llllll}
\hline Dataset & \#Green PSMs & \#Orange PSMs & \#Red PSMs & Total & LIPR (avg \%) \\
\hline SS $_{1}$ & 3858 & 6507 & 156,926 & 167,291 & 61.7 \\
SS $_{2}$ & 62,575 & 6793 & 97,923 & 167,291 & 19.44 \\
\hline
\end{tabular}


classification of the PSMs and the second one, called LIPR, represents the ratio of common peaks that disagree in terms of sequence. In summary, all three indicators show that Strategy 2 outperforms Strategy1. Note that the fact that Strategy2 outperforms Strategy1 could seem obvious at first sight, following the idea that realigning the peaks (as done in Strategy2) could only lead to better results. However, since Strategy 2 naturally widens the search space for each bait spectrum-since it tries to explain $\Delta m$ at different locations in the hit spectra-, this may actually lead to numerous erroneous PSMs. Hence, the fact that Strategy2 behaves better than Strategy1 (with respect to the FDR in this case) was not necessarily easily predictable.

The performances obtained by Strategy 2 also lead us to conclude that, among the two, Strategy 2 is the one that should be implemented in OMS software. Spectral Alignment [5] was already based on a score involving $\Delta m$ to select the best PSM by using, similarly to Strategy2, a realignment process. However, it should be noted that Strategy2 requires to realign very efficiently pairs of spectra if one wants to maintain fast execution time. More recently, MODPlus [16] and Open-pFind [15] select the set of candidate PSMs with sequence tags and then perform realignments. ANN-SoLo [10, 11] and the Hybrid search [9] approximate the improved score obtained taking modified peaks into account (ANN-SoLo's shifted dot product, hybrid search's cosine similarity). On the other hand, some OMS methods still rely on Strategy1 (e.g. MSFragger [12], MetaMorpheus [13]) and do not take $\Delta m$ into account to choose the best candidate PSM-although they may use it once the best candidate is chosen in order to locate the modification. Concerning our color classification, we saw that although Strategy2 recruits more Green and Orange PSMs than Strategy1, it contains proportionally more Red PSMs. However, the average LIPR from the Red class obtained by Strategy 2 is much lower than for Strategy1. This leads us to think that a proportion of the Red PSMs from Strategy2, that share enough peaks corresponding to common subsequences, could be considered as "almost valid" PSMs. More precisely, we believe that with additional methodological and computational effort, some of these Red PSMs could be transfered to the Orange or Green category, an effort that methods implementing Strategy2 should pursue.

We have conducted our study in a "strict" environment, namely comparing theoretical spectra only, and using a score (SPC) that only takes common peaks into account. Although this could be seen as a limitation, it can also be argued that comparing theoretical spectra is an opportunity to provide an overview of the proximity of tryptic peptides extracted from the human proteome from a mass spectrometry point of view. Besides, we place ourselves in an "adversary" context, where no redundancy is allowed, and thus where no PSM corresponds to an exact sequence matching. Concerning SPC, it can be noted that it is, in any method, systematically taken into account at some level in the scoring function. In our case, SPC allowed us to be context and data independent: we consider having no particular knowledge of the dataset nor the spectra at hand, and conducted our experiments in an "unsupervised" manner. Moreover, Strategy2 (as implemented here) always determines the exact value for our score, whereas other OMS methods use approximate scores, slightly surestimating them, which may have an impact on the results. 
By comparing two OMS strategies with theoretical peptides and new indicators, we also developped an environment which allowed us to see and understand elements that are more difficult to see in an experimental context. This protocol could be used to understand principles that are at the heart of other (OMS) MS identification tools, perceive their strengths and weaknesses, in order to configure and calibrate them. For example, the idea behind the color classification is not specific to the SpecOMS software we used nor to the mass shifting implied by Strategy2, and could help assessing the efficiency of any OMS research tool. Furthermore, we believe that our two indicators (used here in a context where both spectra are theoretical) can be adapted-to some extentto classical MS/MS experiments in which theoretical spectra need to be compared to experimental spectra.

Our study also calls for several possible perspectives and extensions, which we briefly mention here: apply our study to experimental contexts (which would require to adapt LIPR and color classification), use a more elaborate score than SPC, improve our color classification algorithm so as to "explain" more PSMs from the current Red class, allow more than one location to explain $\Delta m$, and finally use other types of decoy database. Any combination of the above suggestions could also be considered.

\section{Conclusion}

Despite recent progress, much remains to be done concerning the identification of MS/ MS spectra carrying modifications, and the quality evaluation of OMS methods. The OMS methods present different strategies to identify the best PSM and, in this paper, we compared two such strategies that are well represented in the recent methods. We led this study in a strictly identical environment for both strategies by implementing them in the same software (SpecOMS [14]) and on an ideal dataset formed by theoretical spectra only, that we compare to itself, in order to get rid of identification difficulties [22] due to the imperfection of experimental spectra (noise, missing peaks, etc.). In addition to the conventional FDR, we introduced two new criteria to evaluate the quality of PSMs. For each of the observed criteria, Strategy2, which attempts to align $\Delta m$ between spectra before choosing the best candidate PSM, has always proven to be better. Overall, Strategy2 promotes the PSMs where the hit is derived from the bait by easily identifiable editing operations. Then, more often, the sequence of the bait can be properly determined with Strategy2.

We can note that, regardless of the origin, a modification has the same impact on spectra and therefore, even though we did not perform the comparison on experimental spectra, we can extrapolate the superiority of Strategy 2 in the analysis of any experimental dataset. According to these results, OMS methods should implement Strategy2 but we have to emphasize that Strategy 2 requires an efficient algorithm to find the best alignment of spectra on a very large number of candidate PSMs, the likely reason why it is not done in all OMS methods.

We can also note that OMS methods further complicate the protein inference problem $[23,24]$, since the bait peptide should not be considered as known until $\Delta m$ is explained. Typically, the bait peptides with unexplained $\Delta m$ are those marked as Red in our classification. This confirms the need to investigate more deeply the Red PSMs, by providing an automatic method retrieving bait from hit in these PSMs. Generally, we showed that 
the color classification and also the LIPR are pertinent indicators that can be considered independently of the FDR. Then, with some additionnal efforts, it would be interesting to adapt these criteria to usual MS/MS experiments in which theoretical spectra need to be compared to experimental spectra.

\section{Methods}

\section{Peptide identification using SpecOMS}

We implemented two strategies to find the best PSMs using the SpecOMS software [14]. Next, we applied these two strategies to compare the large set of theoretical spectra generated from the human database against themselves. To perform this comparison between theoretical spectra, we added a new functionality to SpecOMS (in the form of a new search mode).

A peptide that plays the role of an experimental spectrum in a peptide spectrum match (PSM) is called the bait, whereas a peptide associated to a bait in a PSM is called a hit. Parameters were set in such a way that SpecOMS extracted from its data structure SpecTrees [25] all pairs of spectra of the form (bait,hit) whose shared peaks count (SPC) is greater than or equal to 7 .

The above threshold of 7 appears to be a good trade-off for our study: first, it should not be too low so as to avoid many PSMs with a relationship carrying little information between the hit and the bait. It should not be too high either, so as to prevent the case where a low scored candidate PSM would be (definitely) discarded, since the score for such PSMs may be greatly increased (and thus may become chosen as best PSM) by our shifting procedure in Strategy2 (see Fig. 6).

The following parameters of SpecOMS were then set in all the runs: threshold $=7$ (as discussed above), single_match $=$ true, nbMissCleavage $=0$, minimumPeptideLength $=7$, maximumPeptideLength $=30$, maxMassesCount $=60$, minimumScore $=60$, decoyBase $=$ true. Some of these parameters (and in particular minimumScore) were set so as to force SpecOMS not to search for missed cleavages nor semi-tryptic peptides: since we compare a set of peptides to itself, we know these phenomena cannot appear.

Depending on the run, the parameter "shift" of SpecOMS was set to false (Strategy1) or true (Strategy2). More precisely, in Strategy1, for each bait, SpecOMS selects the best PSM based on the highest SPC, a score that we call raw SPC.

In Strategy2, the best PSM for a given bait $b$ is selected after the following two-step procedure is applied: first, for every candidate hit $h$ for $b$ such that $\Delta m \neq 0$, SpecOMS realigns $h$ to $b$ by shifting its masses (by $\Delta m$ ) at each possible relevant location in the spectrum, and retains the shift location in $h$ that yields the best newly computed SPC (a principle frequently used to explain $\Delta m$ [26]). Second, SpecOMS chooses the best PSM among the candidate PSMs for $b$, based on the newly computed SPC, that we call shift SPC. See Figs. 6 and 7 for an illustration.

\section{Data}

The human proteome was downloaded from Ensembl 99, release GrCh38 [27] on the Ensembl FTP server. Proteins predicted with the annotation "protein coding" were added to 116 contaminant proteins downloaded from the cRAP contaminant database. 

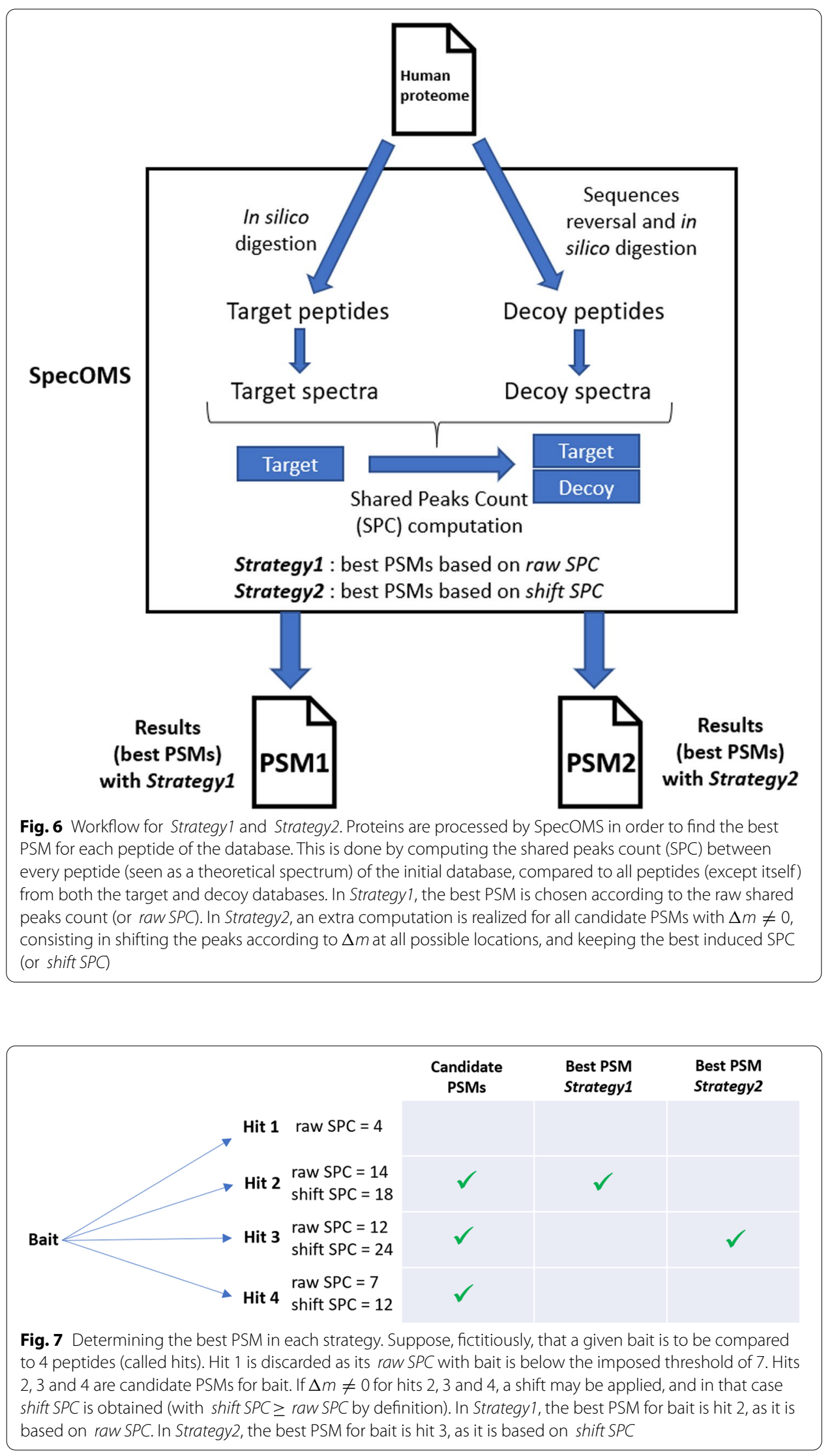
The resulting set of proteins is referred to as the target database. After in silico digestion by trypsin, which cleaves after lysine and arginine ( $\mathrm{K}$ and $\mathrm{R}$ ), peptides whose lengths are out of the range 7 to 30 amino acids (inclusive), together with those containing the letter ' $\mathrm{X}$ ' (representing an unknown amino acid-about 3\%) were removed. SpecOMS generated the decoy database from the target database by reversing the initial protein sequences before the in silico digestion is applied.

\section{Theoretical spectra generation}

Each peptide is fragmented in silico by SpecOMS, so as to transform it into a theoretical spectrum. For this, ions from the $b$ and $y$ series are generated, each with the same intensity unit, as intensity is not taken into account in our study (only the shared peaks count (SPC), i.e. the number of common peaks - or masses-will be considered). For a given peptide, the set of generated masses represents its theoretical spectrum.

\section{Measuring the quality of PSMs}

Different measures were performed on the two datasets obtained with Strategy1 or Strategy2, in particular to determine to which extent the chosen identification strategy impacts the results.

The first classical measure that we used is the number of PSMs we can validate at a given False Discovery Rate (FDR). We calculated the FDR as the proportion of best PSMs of the form (bait,hit) for which the hit is a decoy, over the total number of best PSMs. In this work, we were essentially interested by PSMs for which the FDR is less than $1 \%$.

\section{The Green/Orange/Red classification}

Another parameter which we consider as informative for validating MS/MS results, notably in this context, is our ability to explain a PSM of the form (bait,hit) obtained by a given strategy ; by "explain", we mean unambiguously determine the transformation (in terms of amino acid sequence) that is required to retrieve the bait starting from the hit. Recall that since distinct pairwise peptides are compared, bait and hit necessarily differ in terms of sequence ; besides, they cannot differ otherwise (e.g. due to chemical modifications) since our set is composed of theoretical spectra only. Thus, the question we ask ourselves is the following: given a PSM (bait,hit) together with $\triangle m$, shift SPC and its correspondng best shift location, how difficult is it to precisely explain bait from hit? For this, we introduce here a classification of PSMs into three colors (Green, Orange or Red), depending on this level of difficulty, from the easiest (Green) to the hardest (Red). In a nutshell, Green means that we are able to explain the link between hit and bait unambiguously, Orange contains some level of ambiguity, and Red means that further information and/or computational efforts are necessary to explain the relationship between bait and hit. For instance, if $\Delta m$ is explained by a single insertion, deletion or substitution, the corresponding PSM will be classified as Green, whereas if several consecutive insertions, deletions and/or substitutions are necessary at a given location, it will be classified as Orange since the location is known, but the sequence order is not completely determined (e.g., if we know that amino acids $\mathrm{A}$ and $\mathrm{L}$ need to be inserted at a given location, some ambiguity remains since we can either insert "AL" or "LA"). Finally, a PSM is Red whenever it is neither Green or Orange. Red thus represents either valid identifications 
that are too difficult to explain (e.g., when bait and hit differ at two or more distinct locations), or invalid ones (e.g., when $\Delta m=0$, while bait and hit have very dissimilar sequences). Algorithm 1 describes our classification algorithm in details, and we also refer to Fig. 3 for an illustration on different examples.

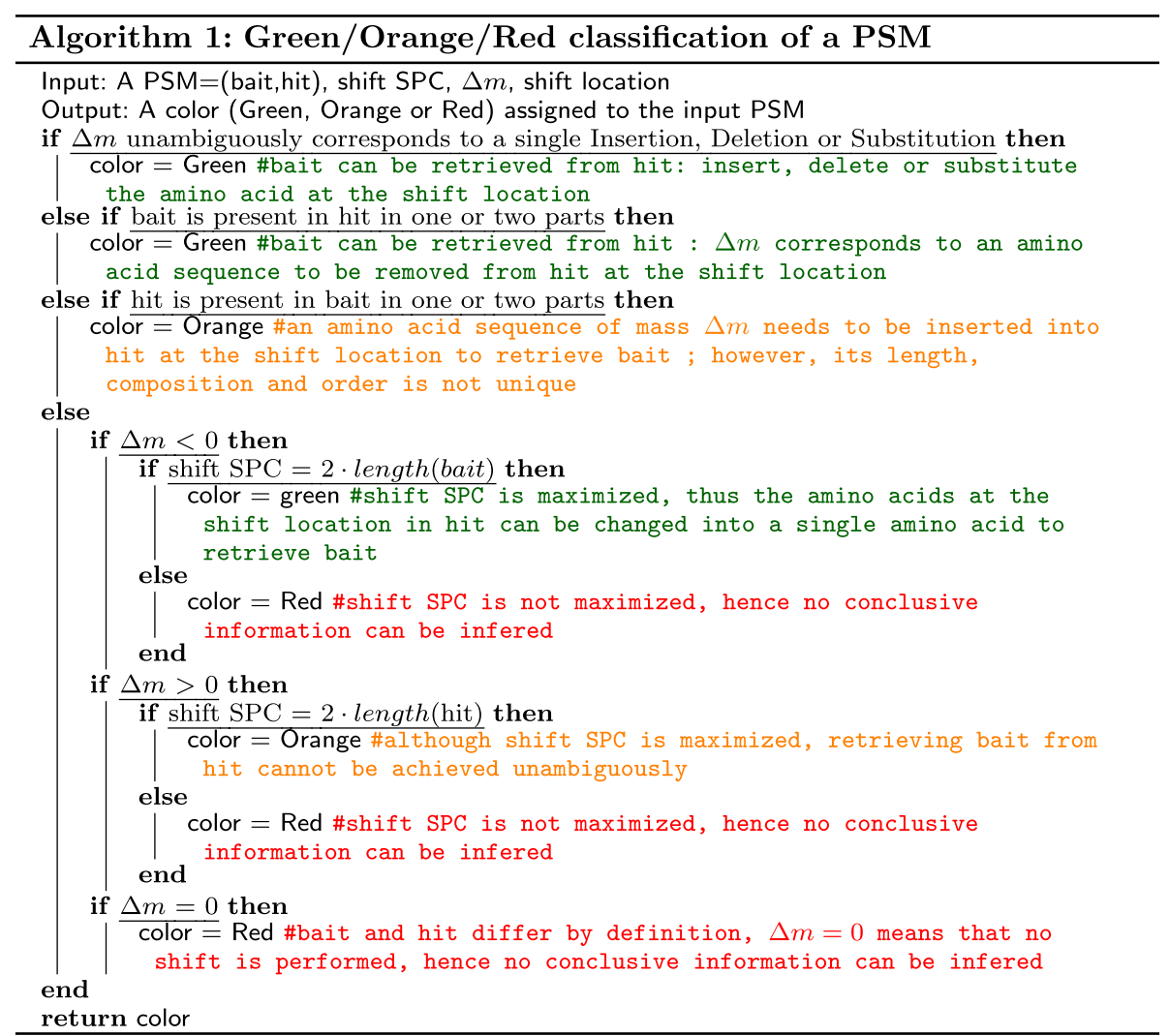

\section{Low Information Peaks Rate (LIPR)}

In an MS/MS experiment, spectra are considered similar to each other if they share a high number of masses. Ions from the same series (i.e., $y$-ions or $b$-ions in our case), which represent the same fragment, necessarily possess the same mass. Consequently, common masses represent relevant information concerning sequence similarity. However, the converse is not always true: identical masses may not represent identical sequences, for example when amino acids are permuted (e.g., AEAE and EEAA have the same mass) or in more complex situations when combinations of different amino acids turn out to have the same total mass (e.g., KE and GVT have the same mass).

Following the above discussion, we introduce here a new measure, that we call Low Information Peaks Rate (or LIPR), which is defined as follows: for a given PSM (bait,hit), LIPR(bait,hit) is the ratio of common masses between bait and hit that do not correspond to identical sequences (see Fig. 8 for an illustration). LIPR is thus a value between 0 and 1 (which we will express as a percentage) ; a LIPR close to 0 implies that the two amino acid sequences of bait and hit are very similar. In that case, one can argue that the PSM at hand 


\begin{tabular}{|c|c|c|c|c|c|}
\hline \multicolumn{2}{|c|}{ SISSINR / SISQIESINR } & \multicolumn{2}{|c|}{ NEDIIQSIQR / NDEIMVSIQR } & \multicolumn{2}{|c|}{ TEGIPIGIIAHGK / EASIPIGIIVVR } \\
\hline Masses & Sequences & Masses & Sequences & Masses & Sequences \\
\hline 88.0393 & $S<--->S \checkmark$ & 115.0502 & $N<--->N \checkmark$ & 288.119 & TEG $<--->$ EAS $x$ \\
\hline 201.1234 & $\mathrm{SI}<--->\mathrm{SI} \checkmark$ & 359.1197 & NED $<-->$ NDE $x$ & 401.2031 & TEGI <---> EASI $x$ \\
\hline 288.1554 & SIS $<--->$ SIS $\checkmark$ & 472.2038 & NEDI $<--->N D E I x$ & 498.2558 & TEGIP <---> EASIP $x$ \\
\hline 175.119 & $R<--->R \checkmark$ & 175.119 & $R<--->R \checkmark$ & 611.3399 & TEGIPI <---> EASIPI $x$ \\
\hline 289.1619 & $\mathrm{RN}<--->\mathrm{RN} \checkmark$ & 303.1775 & $R Q<--->R Q \checkmark$ & 668.3614 & TEGIPIG $<--->$ EASIPIG $x$ \\
\hline 402.2459 & RNI $<--->$ RNI $\checkmark$ & 416.2616 & RQI $<-->$ RQI $\checkmark$ & 781.4454 & TEGIPIGI <---> EASIPIGI $x$ \\
\hline 489.278 & $\begin{array}{l}\text { RNIS <---> RNIS } \checkmark \\
\text { a }\end{array}$ & 503.2936 & $\begin{array}{l}\text { RQIS <---> RQIS } \\
\text { b }\end{array}$ & 894.5295 & $\begin{array}{l}\text { TEGIPIG } \|<-->\text { EASIPIG } \| x \\
\text { C }\end{array}$ \\
\hline
\end{tabular}

Fig. 8 Low Information Peaks Rate (LIPR) for 3 different PSMs. Masses are in Dalton, and the "Sequences" column in each example represents all fragmented peptides having the same mass for the considered PSM. A green check symbol denotes similar sequences, whereas a red cross denotes distinct sequences. a No common masses corresponds to distinct amino acid sequences, thus the LIPR for this PSM is equal to $0 \%$; $\mathbf{b}$ 2 common masses over a total of 7 correspond to distinct sequences, thus the LIPR for this PSM is equal to $\frac{2}{7}=28.57 \%$; c all common masses in this PSM correspond to distinct sequences, hence the LIPR for this PSM is equal to $100 \%$

is relevant, and that retrieving bait from hit may be feasible. On the other hand, when LIPR is close to 1 , both sequences, although sharing a non negligible number of masses, represent very dissimilar sequences, and the PSM can thus be considered as debatable.

Abbreviations

FDR: False discovery rate; LIPR: Low information peaks rate; MS: Mass spectrometry; OMS: Open Modification Search; PSM: Peptide spectrum match; PTM: Post-translational modification; SPC: Shared peaks count.

\section{Acknowledgements}

Not applicable.

\section{About this supplement}

This article has been published as part of BMC Bioinformatics Volume 22, Supplement 2 2021: 15th and 16th International Conference on Computational Intelligence methods for Bioinformatics and Biostatistics (CIBB 2018-19). The full contents of the supplement are available at https://bmcbioinformatics.biomedcentral.com/articles/supplements/volum e-22-supplement-2.

\section{Authors' contributions}

All the authors designed and conceived the study; AL conducted the experiments; AL, DT, JG and GF analyzed the results. AL, DT and GF wrote the manuscript. All the authors read and approved the final manuscript.

\section{Funding}

Supported by the French National Research Agency (ANR-18-CE45-004), ANR DeepProt.

\section{Availability of data and materials}

The human proteome was downloaded from Ensembl 99, release GrCh38 on the Ensembl FTP server ftp://ftp.ensem bl.org/pub/release-99/fasta/homo sapiens/pep/. Proteins predicted with the annotation "protein coding" were added to 116 contaminant proteins downloaded from the CRAP contaminant database ftp://ftp.thegpm.org/fasta/cRAP. The SpecOMS software is available at https://github.com/dominique-tessier/SpecOMS.

Ethics approval and consent to participate

Not applicable.

Consent for publication

Not applicable.

\section{Competing interests}

The authors declare that they have no competing interests.

\section{Author details}

1 CNRS, LS2N, Université de Nantes, 44000 Nantes, France. ${ }^{2}$ BIBS Facility, INRAE, 44316 Nantes, France. ${ }^{3}$ UR BIA, INRAE, 44316 Nantes, France.

Received: 16 December 2020 Accepted: 8 January 2021 Published: 26 April 2021 


\section{References}

1. Prabakaran S, Lippens G, Steen H, Gunawardena J. Post-translational modification: nature's escape from genetic imprisonment and the basis for dynamic information encoding. Wiley Interdiscip Rev Syst Biol Med. 2012;4(6):565-83.

2. Smith LM, Kelleher NL, Consortium for Top Down Proteomics. Proteoform: a single term describing protein complexity. Nat Methods. 2013;10(3):186-7.

3. Griss J, Perez-Riverol Y, Lewis S, Tabb DL, Dianes JA, Del-Toro N, et al. Recognizing millions of consistently unidentified spectra across hundreds of shotgun proteomics datasets. Nat Methods. 2016;13(8):651-6.

4. Chick JM, Kolippakkam D, Nusinow DP, Zhai B, Rad R, Huttlin EL, et al. An ultra-tolerant database search reveals that a myriad of modified peptides contributes to unassigned spectra in shotgun proteomics. Nat Biotechnol. 2015:33(7):743-9.

5. Tsur D, Tanner S, Zandi E, Bafna V, Pevzner PA. Identification of post-translational modifications via blind search of mass-spectra. In: Proceedings IEEE computational systems bioinformatics conference. 2005; p. 157-66.

6. Searle BC, Dasari S, Wilmarth PA, Turner M, Reddy AP, David LL, et al. Identification of protein modifications using MS/ MS de novo sequencing and the OpenSea alignment algorithm. J Proteome Res. 2005;4(2):546-54.

7. Elias JE, Gygi SP. Target-decoy search strategy for increased confidence in large-scale protein identifications by mass spectrometry. Nat Methods. 2007;4(3):207-14.

8. Horlacher $\mathrm{O}$, Lisacek F, Müller M. Mining large scale tandem mass spectrometry data for protein modifications using spectral libraries. J Proteome Res. 2016;15(3):721-31.

9. Burke MC, Mirokhin YA, Tchekhovskoi DV, Markey SP, Heidbrink Thompson J, Larkin C, et al. The hybrid search: a mass spectral library search method for discovery of modifications in proteomics. J Proteome Res. 2017;16(5):1924-35.

10. Bittremieux W, Meysman P, Noble WS, Laukens K. Fast open modification spectral library searching through approximate nearest neighbor indexing. J Proteome Res. 2018;17(10):3463-74.

11. Bittremieux W, Laukens K, Noble WS. Extremely fast and accurate open modification spectral library searching of high-resolution mass spectra using feature hashing and graphics processing units. J Proteome Res. 2019;18(10):3792-9.

12. Kong AT, Leprevost FV, Avtonomov DM, Mellacheruvu D, Nesvizhskii Al. MSFragger: ultrafast and comprehensive peptide identification in mass spectrometry-based proteomics. Nat Methods. 2017;14(5):513-20.

13. Solntsev SK, Shortreed MR, Frey BL, Smith LM. Enhanced global post-translational modification discovery with MetaMorpheus. J Proteome Res. 2018;17(5):1844-51.

14. David M, Fertin G, Rogniaux H, Tessier D. SpecOMS: a full open modification search method performing all-toall spectra comparisons within minutes. J Proteome Res. 2017;16(8):3030-8. https://doi.org/10.1021/acs.jprot eome.7b00308.

15. Chi H, Liu C, Yang H, Zeng WF, Wu L, Zhou WJ, et al. Comprehensive identification of peptides in tandem mass spectra using an efficient open search engine. Nat Biotechnol. 2018:36:1059-61.

16. Na S, Kim J, Paek E. MODplus: robust and unrestrictive identification of post-translational modifications using mass spectrometry. Anal Chem. 2019;91(17):11324-33.

17. Devabhaktuni A, Lin S, Zhang L, Swaminathan K, Gonzalez CG, Olsson N, et al. TagGraph reveals vast protein modification landscapes from large tandem mass spectrometry datasets. Nat Biotechnol. 2019;37(4):469-79.

18. Tabb DL, Murugan BD, Okendo J, Nair O, Blackburn JM, Buthelezi SG, et al. Open search unveils modification patterns in formalin-fixed, paraffin-embedded thermo HCD and SCIEX TripleTOF shotgun proteomes. Int J Mass Spectrom. 2020;448:116266

19. Noble WS. Mass spectrometrists should search only for peptides they care about. Nat Methods. 2015;12(7):605-8.

20. Sticker A, Martens L, Clement L. Mass spectrometrists should search for all peptides, but assess only the ones they care about. Nat Methods. 2017;14(7):643-4.

21. Fertin G, David M, Rogniaux H, Tessier DT. MS/MS spectra interpretation and the interest of SpecFit for identifying uncommon modifications. In: Proceedings 16th international conference on computational intelligence methods for bioinformatics (CIBB'19). LNBI. Springer; 2020.

22. Kim MS, Zhong J, Pandey A. Common errors in mass spectrometry-based analysis of post-translational modifications. Proteomics. 2016;16(5):700-14.

23. Nesvizhskii Al, Aebersold R. Interpretation of shotgun proteomic data: the protein inference problem. Mol Cell Proteomics. 2005;4(10):1419-40.

24. Huang T, Wang J, Yu W, He Z. Protein inference: a review. Brief Bioinform. 2012;13(5):586-614.

25. David M, Fertin G, Tessier D. SpecTrees: an efficient without a priori data structure for MS/MS spectra identification In: Frith M, Storm Pedersen CN, editors. Algorithms in bioinformatics. Lecture notes in computer science. Cham: Springer International Publishing; 2016. p. 65-76.

26. Ahrné E, Müller M, Lisacek F. Unrestricted identification of modified proteins using MS/MS. Proteomics. 2010;10(4):671-86.

27. Yates AD, Achuthan P, Akanni W, Allen J, Allen J, Alvarez-Jarreta J, et al. Ensembl 2020. Nucleic Acids Res. 2020;48(D1):D682-8.

\section{Publisher's Note}

Springer Nature remains neutral with regard to jurisdictional claims in published maps and institutional affiliations. 